\title{
APPLICATION OF NEURO-FUZZY THRESHOLDING TO BRIDGE PAINTING DEFECT RECOGNITION
}

\author{
Po-Han Chen ${ }^{1}$ and Luh-Maan Chang ${ }^{2}$ \\ ${ }^{1}$ Ph.D. Candidate, Div. of Constr. Eng. and Mgmt., School of Civil Eng., \\ Purdue Univ.,West Lafayette, IN 47907,USA.E-mail: pohan@purdue.edu \\ ${ }^{2}$ Associate Professor, Div. of Constr. Eng. and Mgmt., School of Civil Eng., \\ Purdue Univ., West Lafayette, IN 47907, USA.
}

\begin{abstract}
The neuro-fuzzy thresholding method segments a grayscale image into three areas in accordance with the illumination values of the pixels in the image. The three average illumination values of the three areas are then sent to a pre-trained neural network to obtain the three corresponding threshold values. Fuzzy theory is used to adjust the gray level values of the pixels along the boundaries between areas. Finally, image thresholding is applied to get a binary image containing only the object pixels and the background pixels.
\end{abstract}

Keywords: neural networks, fuzzy adjustment, image thresholding, k-means algorithm, neurofuzzy thresholding.

\section{INTRODUCTION}

As the computerized technologies were widely utilized, the digital image processing was also prevalently adopted in lots of industries [1]. In the construction area, the image processing has been used for defect recognition on steel bridge painting and underground sewer systems $[2,3]$. However, nonuniformly illuminated images always cause recognition problems and affect the accuracy.

In order to resolve these problems, the neurofuzzy thresholding approach was proposed. This approach segments an image into three areas based on illumination and conducts area-based thresholding. The neural network is used in this approach for automatic generation of three threshold values, with the three average illumination values of the three areas as the input. The fuzzy theory is utilized to smooth and adjust the gray level values of the image pixels along the boundaries.

The framework of the neuro-fuzzy thresholding approach, including the methodology of this approach, the training of the neural network, and the rationale of the fuzzy adjustment, will be clarified in this paper.

\section{BACKGROUND}

\subsection{Neural Networks}

A neural network, which contains several layers, is constituted with a number of artificial neurons. According to Tsoukalas and Uhrig, a neural network can be defined as [4]:

" A data processing system consisting of

a large number of simple, highly interconnected processing elements (artificial neurons) in an architecture inspired by the structure of the cerebral cortex of the brain."

In practice, three-layered feedforward neural networks are the mostly utilized multi-layer neural networks. "Feedforward" means no lateral connections exist between the artificial neurons in a given layer and the information flow does not go back to previous layers. Figure 1 shows the structure of a neural network.

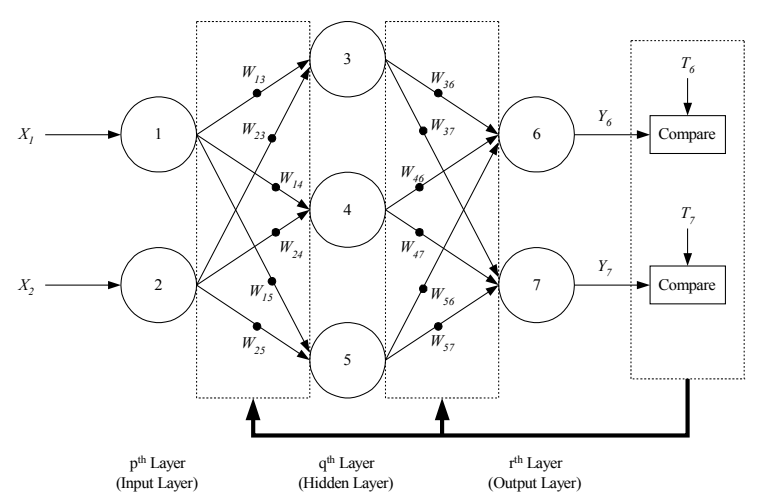

Figure 1. Structure of a Neural Network 
There are three different layers in the structure of neural networks: the input layer, the hidden layer, and the output layer. The input layer is the incoming layer of a neural network that receives information, and the output layer is the outgoing layer of a neural network that sends out filtered results. The hidden layer is referred to the layer (or the layers) between the input layer and the output layer. The outputs of a neural network will be compared to the targets for adjustment of the weights. This process is called "training" [4,5].

\subsection{Fuzzy Systems}

Fuzzy theory deals with relative importance, which coincides with general human intuition. For example, in a hot sunny day, people can feel it is hot, but cannot tell how hot it is in terms of degrees. This is the feature of fuzzy systems, which convert linguistic expressions to numerical and analytical forms. A fuzzy system contains several membership functions. Each membership function has the universe of discourse (the collection of all input values) as its $\mathrm{x}$-axis, and the membership values (the occurrence probabilities of input values) as its y-axis. The operation of fuzzy systems is controlled by a set of If-Then rules and an implication method. The Mamdani implication method is the most frequent one. With these If-Then rules and the implication method, fuzzy systems are capable of mapping an input (or inputs) to an appropriate output $[4,6]$. Figure 2 shows a fuzzy system. In this system, the universes of discourse of all the three membership functions range from 0 to 20 .

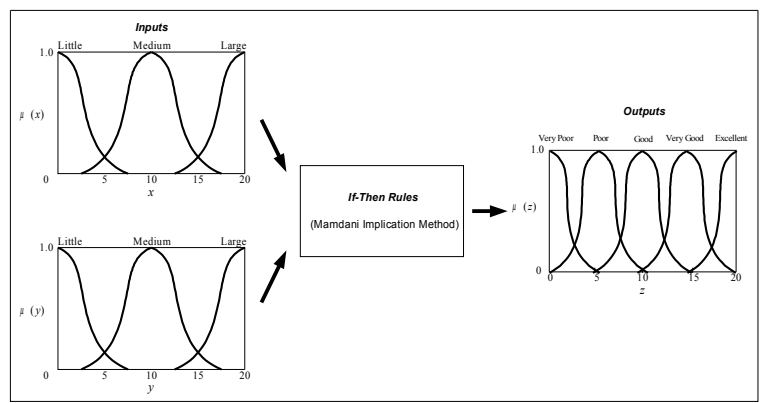

Figure 2. A Fuzzy System

\subsection{K-Means Algorithm}

The k-means algorithm, which divides a group of samples based on the distance to the cluster means, is the simplest clustering method. To apply the k-means algorithm, the feature vector of each sample and the number of clusters should be determined in advance. Also, the number of clusters should be no more than the number of samples. In general, the k-means algorithm has the following steps $[2,7]$ :

1. Randomly assign $K$ samples' feature vectors as the first $K$ means $\left(M^{(1)}{ }_{i}, i\right.$ $=1$ to $K$, and ${ }^{(1)}$ indicates the first iteration) of the $K$ clusters.
2. Assign the remaining $(N-K)$ samples to the closest clusters based on the distance between each sample and each cluster mean, where $N$ is the total number of samples.

3. Re-compute the mean of each new cluster $\left(M^{(2)}{ }_{i}, i=1\right.$ to $K$, and ${ }^{(2)}$ indicates the second iteration).

4. Reassign the $N$ samples to the clusters with the closest means.

5. Repeat $3-4$ until no further change occurs.

\subsection{Image Thresholding}

Image thresholding, which is usually applied to grayscale images, is a method used for image segmentation. The concept of image thresholding is simple. First, select an appropriate threshold value. Then, use the threshold value to segment an image. Pixels with gray level values greater than the threshold value are considered as the background, and pixels with gray level values less than the threshold value are thought of as the object (or the foreground), or vice versa. Pixels with the same value as the threshold can be classified as either the object or the background. Image thresholding can be expressed by Eq. (1):

$$
F(x, y)=\left\{\begin{array}{l}
P_{B}, G(x, y) \geq t \\
P_{O}, G(x, y)<t
\end{array}\right.
$$

where $G(x, y)$ is the gray level function, which maps the pixel located on $(x, y)$ to its corresponding gray level value. $t$ is the threshold value. $F(x, y)$ is the thresholding function that classifies pixels as the background pixels $\left(P_{B}\right)$ or the object pixels $\left(P_{o}\right)$. Image thresholding can also divide an image into $N$ segments. In this case, $(N-1)$ threshold values are needed, and Eq. (1) can be extended as [2,8]:

$F(x, y)=\left\{\begin{array}{ccc}P_{1}, & G(x, y) \geq t_{1} \\ P_{2}, & t_{1}>G(x, y) \geq t_{2} \\ \vdots \\ P_{N}, t_{N-1}>G(x, y)\end{array}\right.$

\section{METHODOLOGY}

The neuro-fuzzy thresholding approach conducts an area-based image processing. Figure 3 illustrates the methodology flow of this approach.

First, the acquired image should be converted to gray scale. The obtained grayscale image will then be sent to an image processing software for illumination-based segmentation. The segmentation divides an image into three areas in accordance with the illumination of the pixels in the image. The three average illumination values of the three areas will be 
collected and input to a pre-trained neural network. The output of the neural network will be three threshold values, which will be used for area-based image thresholding. Meanwhile, the fuzzy theory will be applied to the segmented grayscale image and adjust the gray level values of the pixels on both sides of the boundaries. After the three threshold values are available and the boundary pixels between areas are adjusted with the fuzzy system, the segmented grayscale image will be thresholded with the three obtained threshold values and the defects can be recognized and calculated.

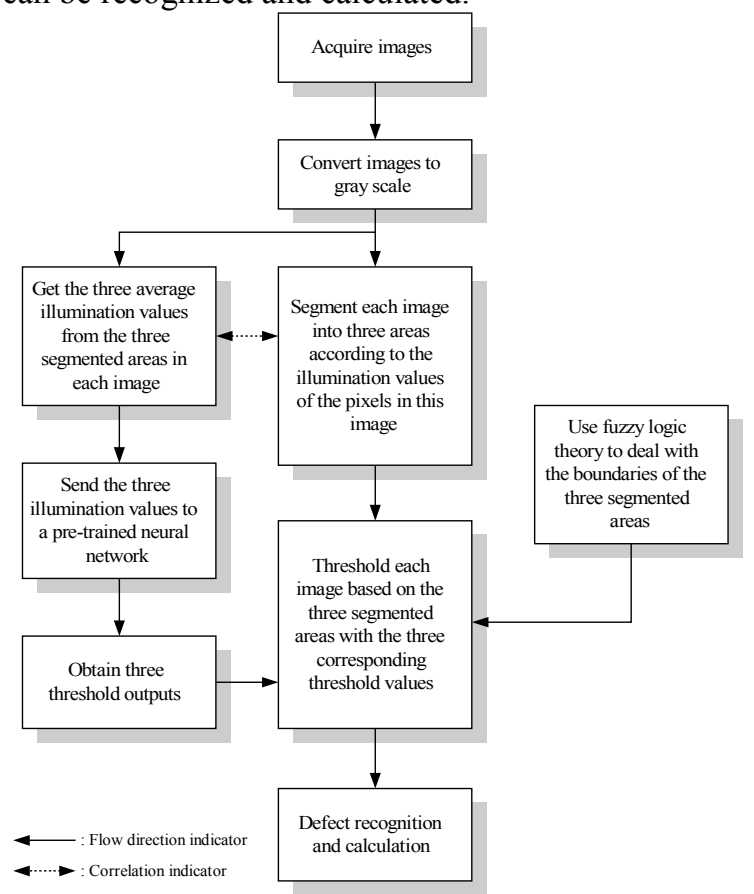

Figure 3. Methodology of the Neuro-Fuzzy Thresholding Approach

\section{TRAINING OF NEURAL NETWORK}

The neural network plays an important role in the neuro-fuzzy thresholding approach. It automatically generates three optimal threshold values for later image thresholding. In this section, the rationale of how the neural network is trained and how to obtain the required training pairs (an input and a target output are called a training pair) is presented.

Figure 4 shows the training process of the neural network. To begin with, a sample grayscale image should be segmented into three areas based on illumination using an appropriate image processing software. The three average illumination values of the three areas will be the input to the neural network.

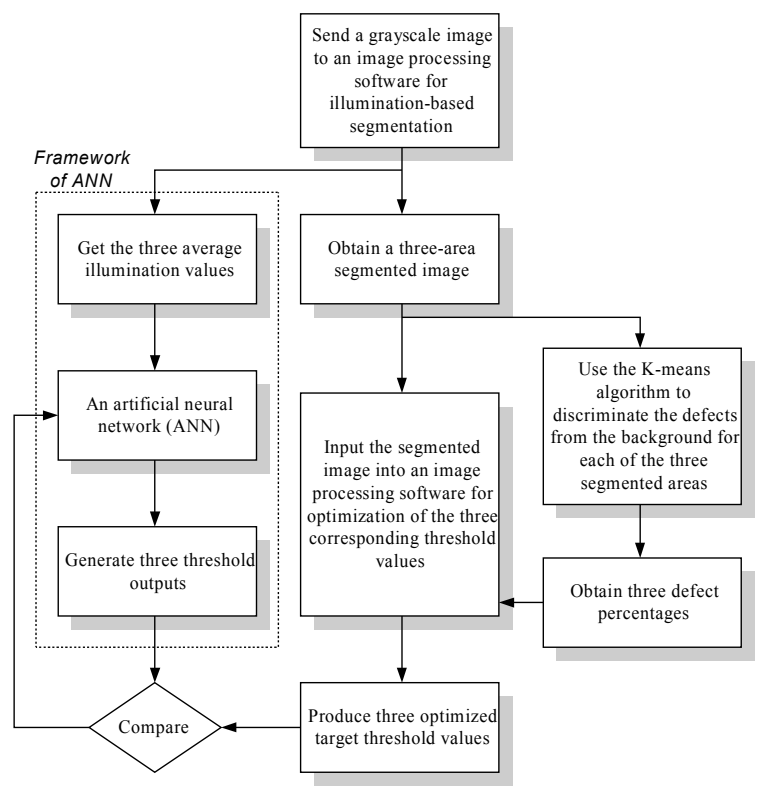

Figure 4. Training Process of the Neural Network

Then, the k-means algorithm will be applied to the segmented image and discriminate the defects (or the objects) from the background for each area. The defect (or object) percentage of each area will be computed. Based on the three defect percentages, the optimal threshold values for the three segmented areas can be acquired by means of an image processing software. The three optimal threshold values are the target values to the neural network and will be compared with the three threshold outputs for further training until the error is acceptably small $[9,10]$.

\section{PROCEDURES OF FUZZY ADJUSTMENT}

The fuzzy adjustment ought to be applied to the image pixels on both sides of the boundaries between areas, as Figure 5 indicates. Figure 6 illustrates the schematic representation of the fuzzy adjustment. Two inputs are included in this fuzzy system, the "positive difference" and the "negative difference." The output is the "gray level adjustment." A set of nine If-Then rules constitutes the kernel of the fuzzy system.

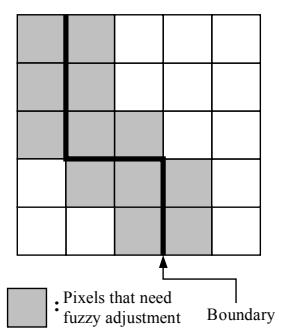

Figure 5. Pixels That Need Fuzzy Adjustment 


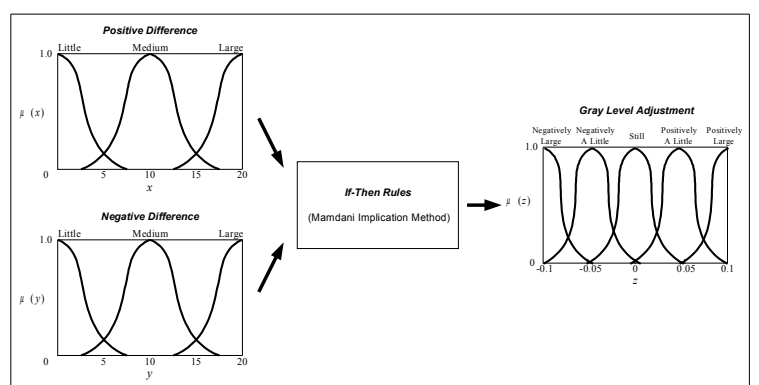

Figure 6. Schematic Representation of Fuzzy Adjustment

For a clear presentation, the fuzzy adjustment system will be broken into three parts, the inputs, the output, and the If-Then rules, for explanation.

\subsection{The Inputs}

Gray level values are the concerns of the fuzzy adjustment. Both the inputs "positive difference" and "negative difference" have three levels: large, medium, and little. The universe of discourse for both inputs ranges from 0 to 20. Differences (both positive and negative) larger than 20 are counted as 20. For an ordinary pixel that needs fuzzy adjustment and is located on $(x, y)$, four neighboring pixels, $(x+1, y), \quad(x-1, y), \quad(x, y+1)$, and $(x, y-1)$, need be considered for difference calculation. In this case, each of the four neighboring pixels has a weight of 1/4 (See Figure 7(a)). For an edge pixel, only three neighboring pixels are considered for difference calculation, and each of them is assigned a weight of 1/3 (See Figure 7(b)). Likewise, for a corner pixel, only two neighboring pixels are considered and each of them has a weight of 1/2 (Figure 7(c)).

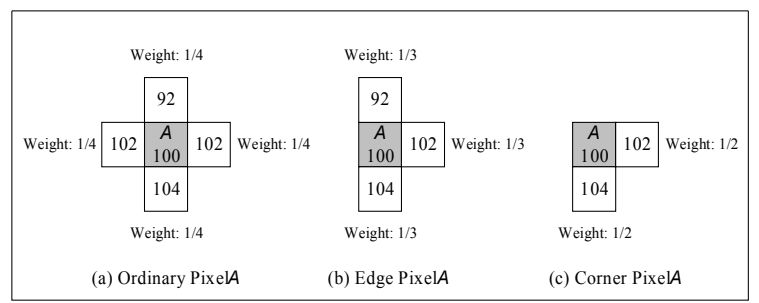

Figure 7. Weighting of Different Pixel Types

The concept of the difference calculation is pretty simple. For an image pixel $A$, the neighboring pixels with gray level values larger than the gray level value of $A$ are the components of the positive difference calculation. Those neighboring pixels with gray level values smaller than the gray level value of $A$ are included in the negative difference calculation. The difference calculation starts with the computation of the gray level difference between pixel $A$ and its neighboring pixels, followed by the multiplication of each gray level difference and its corresponding weight. Finally, all results after multiplication are summed as the gross difference. The calculation of the positive difference and the negative difference should be conducted separately. Figure 8 illustrates the calculation of both positive and negative gray level differences.

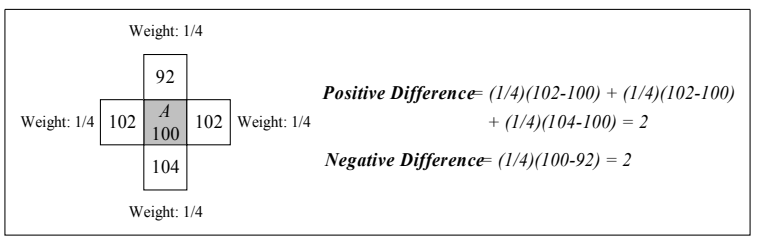

Figure 8. Gray Level Difference Calculation

\subsection{The Output}

The "gray level adjustment" is the output of the fuzzy system. It has values ranging from -0.1 to 0.1 and contains five different levels: negatively large, negatively a little, still, positively a little, and positively large. This means that, in the extreme cases, the gray level value of a pixel to be adjusted could be increased or decreased 10\%. The adjusted gray level value can be expressed by the following equation:

$G_{\text {new }}(x, y)=G_{\text {old }}(x, y) *(1+\beta)$

where $G_{\text {new }}(x, y)$ and $G_{\text {old }}(x, y)$ represent the new gray level value and the old gray level value of the pixel located on $(x, y)$, respectively. $\beta$ is the gray level adjustment amount, which is the output of the fuzzy adjustment system.

\subsection{The If-Then Rules}

There are nine If-Then rules involved in this fuzzy adjustment system. They are listed in Table 1. The Mamdani implication method is used in this system.

Table 1. If-Then Rules for Fuzzy Adjustment

\begin{tabular}{|c|c|c|c|c|c|}
\hline IF & $\begin{array}{c}\text { Positive } \\
\text { Difference }\end{array}$ & AND & $\begin{array}{c}\text { Negative } \\
\text { Difference }\end{array}$ & THEN & $\begin{array}{c}\text { Gray Level } \\
\text { Adjustment }\end{array}$ \\
\hline \multirow{9}{*}{ IF } & Large & \multirow{9}{*}{$A N D$} & Large & \multirow{9}{*}{ THEN } & Still \\
\hline & Large & & Medium & & Positively A Little \\
\hline & Large & & Little & & Positively Large \\
\hline & Medium & & Large & & Negatively A Little \\
\hline & Medium & & Medium & & Still \\
\hline & Medium & & Little & & Positively A Little \\
\hline & Little & & Large & & Negatively Large \\
\hline & Little & & Medium & & Negatively A Little \\
\hline & Little & & Little & & Still \\
\hline
\end{tabular}

\section{STEPWISE NEURO-FUZZY THRESHOLDING MODEL}

The stepwise neuro-fuzzy thresholding model was evolved from the methodology of the neurofuzzy thresholding approach. Therefore, they are basically similar. The purpose of the stepwise neurofuzzy thresholding model is to demonstrate the implementation of this approach in practice. The model contains seven steps, from image acquisition to defect recognition and calculation. Figure 9 illustrates the stepwise neuro-fuzzy thresholding model. 


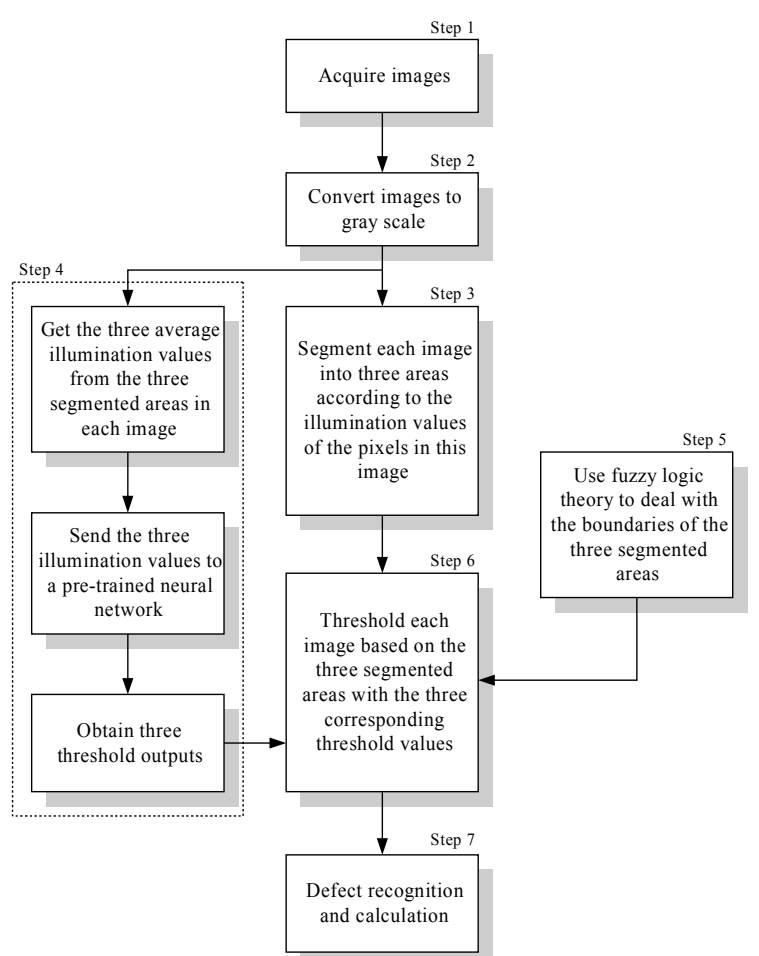

Figure 9. Stepwise Neuro-Fuzzy Thresholding Model

Step 1:

Image acquisition is the first step of the neurofuzzy thresholding model. Image data can be acquired by using a digital camera, and then be transferred to a computer. A steel bridge painting image is used in this case. Figure 10 depicts the image acquisition process.

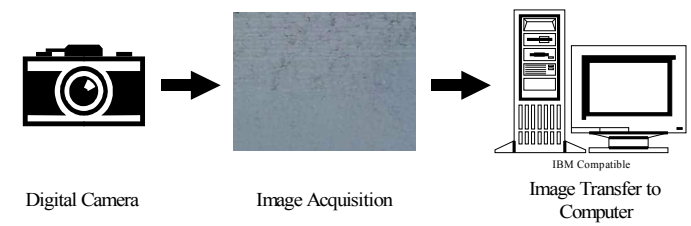

Figure 10. Image Acquisition

Step 2:

The second step is to convert the image to gray scale using an image processing software. In order to process in an efficient and effective way, an image is usually converted to gray scale before processing. Figure 11 demonstrates the process of image conversion to gray scale.

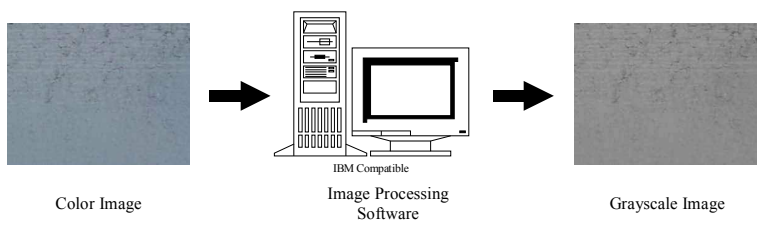

Figure 11. Image Conversion to Gray Scale

Step 3:

After converting the image to gray scale, the illumination value of each pixel can be found. All the pixels in the image are separated into three groups in accordance with their illumination values. Illumination values are between 0 and 1.0 indicates the darkest and 1 indicates and brightest. The average illumination values of the three areas will be computed and serve as the input to a pre-trained neural network. Figure 12 illustrates the illuminationbased image segmentation.

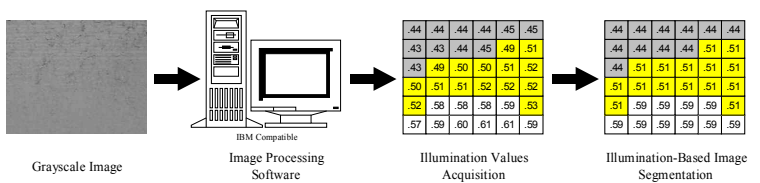

Figure 12. Illumination-Based Image Segmentation

Step 4:

Once the image segmentation is completed, the three average illumination values of the three areas will be sent to a pre-trained neural network to generate three corresponding threshold values, which range from 0 to 255 . The training set for the neural network should be diverse so that the trained neural network will be well rounded and fault tolerant. Figure 13 illustrates the neural computing process of the three threshold values.

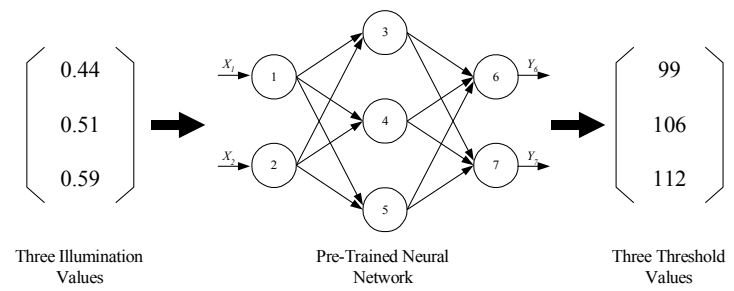

Figure 13. Neural Computing of Threshold Values

Step 5:

In this step, the fuzzy adjustment system is utilized to adjust the gray level values of the image pixels along the boundaries. The gray level adjustment range is from $-10 \%$ to $+10 \%$. Figure 14 shows the flow of fuzzy adjustment.

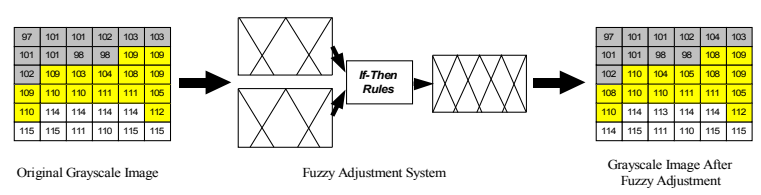

Figure 14. Fuzzy Adjustment on Boundary Pixels

Step 6:

In this step, each area is thresholded according to its corresponding threshold value. Pixels with gray level values smaller than the threshold values (i.e., darker) are considered as the defects (or rusts in this case), and pixels with gray level values larger than the threshold values (i.e., brighter) are considered as the background. Figure 15 depicts the illuminationbased thresholding process. In Figure 15, the values in the grayscale image represent the gray level values of the pixels. The thresholded image is a binary image, with 0's representing the background and 1's representing the defects (rusts). 


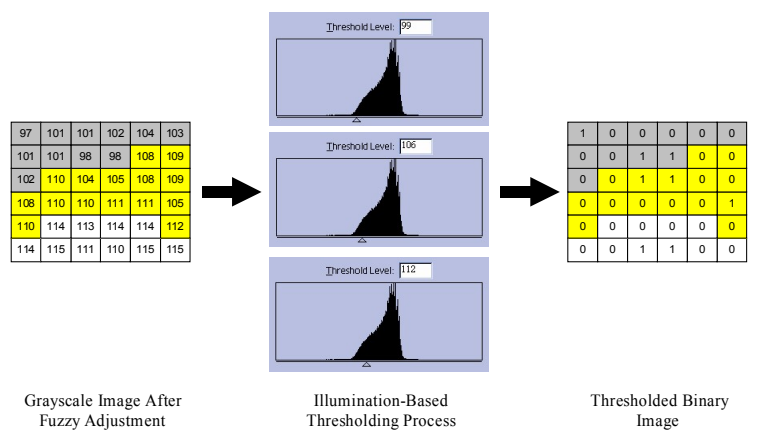

Figure 15. Illumination-Based Thresholding Process

Step 7:

When the thresholding of all the three areas is completed, the defects in the image can be recognized and the defect percentage can be calculated by counting the percentage of the defect pixels out of all the pixels in the image. Figure 16 illustrates the defect recognition and calculation.

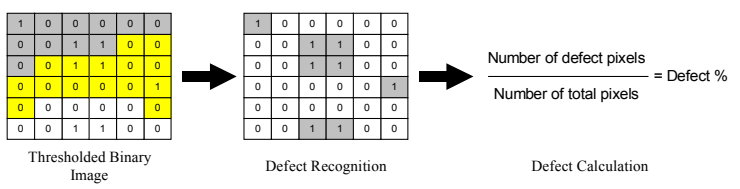

Figure 16. Defect Recognition and Calculation

\section{CONCLUSIONS}

With the prevalence of the computer-related technologies, the digital image processing has been widely used in different industries. In the construction industry, the digital image processing has been experimented on the defect recognition of steel bridge painting. This is great progress and may lead to the automation of steel bridge painting inspection. However, there are still some problems associated with this newly proposed application. Poor quality images remarkably affect the accuracy of this application, and shadows and overillumination are mostly the causes.

In order to resolve these problems, an illumination-based image recognition technique combining the neural network and the fuzzy theory is proposed. The neuro-fuzzy thresholding approach segments an image into three parts in accordance with the illumination of the pixels in the image and thresholds the image based on the three areas. The intelligent learning ability of the neural network is utilized for the automatic generation of three threshold values through the input of the three average illumination values from the three segmented areas. The fuzzy theory is used for smoothing the gray level values of the image pixels along the boundaries.

The proposed neuro-fuzzy thresholding approach is expected to minimize the recognition problems caused by non-uniformly illuminated images and increase the recognition accuracy.

\section{REFERENCES}

[1] Croall, I. F., and Mason, J. P. (1992). Industrial Applications of Neural Networks. Springer-Verlag New York Inc., New York, NY.

[2] AbdelRazig, Y., Chang, L. M., and Skibniewski, M. (1999). "Automated Quality Assessment of Constructed Surfaces Through 'Intelligent' Image Processing." $16^{\text {th }}$ International Symposium on Automation and Robotics in Construction (ISARC), September 22-24, 1999, Madrid, Spain.

[3] Abraham, D., Iseley, T., Prasanth, R., and Wirahadikusumah, R. (1997). "Integrating Sensing Technologies for Underground Utilities Assessment." Infrastructure Condition Assessment: Art, Science, and Practice. Proceedings of the conference sponsored by the Facilities Management Committee of the Urban Transportation Division of the American Society of Civil Engineers, August 25-27, 1997, Boston, MA, 316-325.

[4] Tsoukalas, L. H., and Uhrig, R. E. (1997). Fuzzy and Neural Approaches in Engineering. John Wiley \& Sons, Inc., New York, NY.

[5] Haykin, S. (1999). Neural Networks: A Comprehensive Foundation. Prentice Hall, Upper Saddle River, NJ.

[6] Kosko, B. (1992). Neural Networks and Fuzzy Systems. Prentice-Hall, Inc., Englewood Cliffs, NJ.

[7] De La Blanca, N. (1992). Pattern Recognition and Image Analysis. World Scientific, River Edge, NJ.

[8] Russ, J. C. (1995). The Image Processing Handbook. CRC Press, Inc., New York, NY.

[9] Looney, C. (1997). Pattern Recognition Using Neural Networks. Oxford University Press, New York, NY.

[10] Ripley, B. D. (1996). Pattern Recognition and Neural Networks. Cambridge University Press, New York, NY. 